\title{
Effect of treadmill walking on prefrontal cortex activation and dual-task performance in older adults
}

Chang Yoon Baek ( $\sim$ bcy77@nhimc.or.kr)

National Health Insurance Ilsan Hospital

Hyeong Dong Kim

Korea University

\section{Dong Yup Yoo}

National Health Insurance Ilsan Hospital

\section{Research Article}

Keywords: prefrontal cortex, dual-task, gait, rehabilitation, aging

Posted Date: March 2nd, 2022

DOI: https://doi.org/10.21203/rs.3.rs-1392458/v1

License: (c) This work is licensed under a Creative Commons Attribution 4.0 International License. Read Full License 


\section{Abstract \\ Background}

Older adults have higher prefrontal cortex (PFC) activation and lower dual-task performance than younger adults because of age-related physical and cognitive functional impairments.

\section{Objective}

To investigate the effect of treadmill walking on PFC activation and dual-task performance in older adults.

\section{Methods}

A total of 20 older adults (mean age, $64.35 \pm 2.74$ years) and 20 younger adults (mean age, $30.00 \pm 3.15$ years) performed single- and dual-task walking in overground and treadmill conditions. A wearable functional near-infrared spectroscopy device and gait analyzer were used to analyze PFC activation and dual-task cost (DTC).

\section{Results}

In both groups, treadmill walking demonstrated reduced PFC activation while dual-task walking and reduced DTC compared to overground walking. Furthermore, despite a higher DTC in gait variability, correct response, total response, response index and a higher error score in older adults than in younger adults during overground walking, there was no difference in treadmill walking. The difference in PFC activation between single and dual tasks was also observed only in overground walking. There was no difference in PFC activation between the two age groups.

\section{Conclusions}

Older adults can maintain the similar level of dual-task performance as younger adults in treadmill walking, with PFC activation reduced. However, although older adults recruit PFC level similar to that in younger adults in overground walking, older adults show lower dual-task performance than younger adults due to inefficient use of PFC with aging. This indicates that treadmill and overground walking do not share similar activation patterns during dual-task walking.

\section{Introduction}

Aging leads to degradation of physical and cognitive functions. ${ }^{1}$ Typically, aging-related changes include decreased gait speed, increased gait variability, and executive functional deficits, 2,3 which is correlated with alterations in brain regions, such as loss of frontal-lobe gray matter volume and basal ganglia abnormalities. ${ }^{4,5}$ These negative effects are more easily observed in dual-task performance, on which they exert more influence compared to single-task performance, increasing the associated dual-task interference (DTI). ${ }^{6}$ Hence, dual-task performance could be used to evaluate decreased executive function, decreased gait capacity, or risk of falling with aging. ${ }^{6,7}$ Brain behavioral studies using functional near-infrared spectroscopy (fNIRS) have reported higher prefrontal cortex (PFC) activation during dual-tasking involving executive functions compared to single-tasking. ${ }^{1,8}$ The negative effects of aging (motor and cognition) have caused excessive PFC activation, which was compensatory mechanism indicating over-recruitment of cognitive resources and reduced efficiency of PFC. ${ }^{3,9,10}$ Furthermore, since aging-related changes in the PFC occur rapidly, ${ }^{5}$ we considered that neural mechanisms in dual-task performance may differ between older and younger adults. However, 
some brain imaging studies have shown less PFC activation in older adults during dual-tasking, with condition factors (overground and treadmill walking set-ups) and age considered as factors possibly causing these conflicting findings. ${ }^{1,11,12}$ Although the difference between walking conditions (overground and treadmill) has been raised as a problematic factor affecting the findings of many brain behavioral studies, ${ }^{11,12}$ findings on both treadmill and overground walking have been generalized. ${ }^{13-17}$ To our knowledge, studies addressing walking condition differences and comparing brain activation in older adults to younger adults are lacking. In addition, several studies on dual-task

performance have reported different gait mechanisms for overground and treadmill walking. ${ }^{18,19}$ Treadmill walking led to decreased PFC activation and increased gait stability in patients with Parkinson's disease compared with overground walking. ${ }^{20}$ This is the first study to determine and compare PFC activation and DTC during dual-tasking under different conditions (overground vs. treadmill walking) among younger and older adults. Therefore, we aimed to determine 1) whether the treadmill walking condition leads to lower PFC activation and higher dual-task performance than the overground walking condition and 2) whether older adults have higher PFC activation and lower dual-task performance than younger adults. We hypothesized that 1 ) treadmill walking would lead to lower PFC activation and higher dual-task performance than overground walking, 2) older adults would have higher PFC activation and lower dual-task performance than younger adults, and 3) there would be a difference between older and younger adults on PFC activation and dual-task performance in overground walking, but not in treadmill walking. Therefore, the results of this study will provide an information in designing an appropriate dual-task evaluation and intervention for younger and older adults.

\section{Methods}

\section{Participants}

This was a cross-sectional study in which 40 right-handed adults were recruited and evaluated from April 29 to June 14, 2021. Among them, 20 were older adults (mean age, $64.35 \pm 2.74$ years) and 20 were younger adults (mean age, $30.00 \pm$ 3.15 years). This study was approved by the National Health Insurance Service of the llsan Hospital Institutional Review Board and was conducted in a public hospital in accordance with the Declaration of Helsinki. The study was registered in the Clinical Trial Registry of Korea (https://cris.nih.go.kr; no. KCT0006151, 05/20/2021). All participants provided written informed consent before the evaluation. The inclusion criteria were as follows: 1) adults aged between 20 and 40 years or between 60 and 80 years and 2) no ambulatory assistive devices in daily living activities. The exclusion criteria were as follows: 1) Korean version of the Montreal Cognitive Assessment (K-MoCA) score $\leq 17$, indicating dementia, ${ }^{9} 2$ ) orthopedic disorders; 3) neurological disorders; and 4) poor understanding of the assessment process.

\section{Procedures}

Initially, the K-MoCA was used to evaluate baseline cognitive function. ${ }^{21}$ Additionally, executive function was assessed using the Trail Making Test B (TMTB), in which the time it took for the participant to connect the numbers and letters alternately $(1, A, 2, B, \ldots L, 13)$ was recorded. ${ }^{22}$ Unlike trail making test A, TMTB was associated with dual-task gait ability. ${ }^{23}$ The subjects' gait speed was assessed to measure mobility function with gait analyzer. Especially for older adults, the Short Physical Performance Battery (SPPB), which included gait, sit-to-standing functions, and standing balance, was assessed using scores ranging from 0 to $12 .{ }^{24}$ Furthermore, the Geriatric Depression Scale (GDS) with scores of 0-30 and Falls Efficacy Scale-International (FES-I) with scores of 14-64 were used to assess depression and fear of falls, respectively, ${ }^{25,26}$ which can affect the dual-task performance in older adults (i.e., higher scores indicate negative effects). ${ }^{10}$ Retrospective falls within 6 months were also collected. The subjects' preferred treadmill walking speed was measured first by applying an initial speed of $2.0 \mathrm{~km} / \mathrm{h}$ and adding increments of $0.1 \mathrm{~km} / \mathrm{h}$, then by decreasing the speed from $6.5 \mathrm{~km} / \mathrm{h}$, which was confirmed with previous findings. ${ }^{19}$ For the cognitive task, the subjects 
performed serial subtractions of 7 from 3-digit numbers (i.e., 100 to 200) randomly provided by the assessor for 30 seconds, and the correct response (CR), total response, error response number and response index (the number of $\mathrm{CR} /$ total response $\times 14$ ) were calculated. ${ }^{9,16}$ This task was first performed with the subjects in a seated position before being re-evaluated in overground and treadmill walking.

In evaluating the outcome measures, a dual-task evaluation (i.e., walking with a secondary task) in different conditions was conducted 20 minutes after the cognitive single-task evaluation to avoid any learning effect. The order of the walking conditions for each group was counterbalanced, in which the order of conditions of the second subject was reversed depending on the order of the walking condition performed by the first subject who was applied with randomization. To rule out subject fatigue, which could influence task performance, ${ }^{27}$ a 10 -min break was provided between the different walking conditions, and the order of dual-task and single-task trials was randomized within each walking condition. The gait assessments lasted for $30 \mathrm{~s}$ for each condition. Each subject maintained a quiet position for $30 \mathrm{~s}$ before performing the tasks, and tasks started with the instruction "beginning." In single-task gait, walking proceeded without cognitive tasks, and in dual-task gait, a cognitive task (serial subtractions by 7 ) was performed while walking. The serial subtraction task during walking is widely used as it induces more PFC activation and is related to executive functions. ${ }^{28,29}$ Subjects performed the task(walking + serial subtraction task) at natural walking speed without answering loudly. The subject's response was simultaneously recorded, to which the $\mathrm{CR}$, the total response, response index, and error score (the number of error/CR $\times 100$ ) in each condition were calculated, determining the cognitive task performance. $^{30}$

During all gait tasks, PFC activation and dual-task performance (gait and cognitive task) were measured during overground and treadmill walking.

\section{Assessment of PFC activation}

We used a wearable 48-channel fNIRS device (NIRSIT, OBELAB Inc., Seoul, South Korea), which is specialized for PFC activation measurement (Fig. 1), ${ }^{31}$ compared to the equipment with only 2 to 16 channels used in other studies, ${ }^{9-12,32,33}$ as it covers the overall prefrontal area. ${ }^{34,35}$ We used wavelengths of $780 \mathrm{~nm}$ and $850 \mathrm{~nm}$ with an $8.138-\mathrm{Hz}$ sampling rate, and we set the interoptode distance, including the 24 lasers and 32 detectors, to only $3 \mathrm{~cm}$, which could obtain the greater contribution of the cerebral layer than extracerebral layers. ${ }^{34}$ The differential path length factor (DPF) which was a dimensionless correction factor used in the modified Beer-Lambert law to measure hemodynamic response, was set to 6 and 5.2 for wavelengths, $780 \mathrm{~nm}$ and $850 \mathrm{~nm}$ respectively. In accordance with the localization of the FPz based on the 10-20 international EEG system, this device was placed on the forehead and fixed by an elastic band and calibrated for each subject to eliminate signal saturation provided by biological factors (e.g., skin and hair color, skull thickness). The signal in the prefrontal area was measured during each task. For data processing, the NIRSIT processing package program based on MATLAB (Version 2019b; MathWorks Inc., Natick, Mass., USA) was used, following the recommendations of recent review studies with fNIRS. 8,36 First, to convert the raw data extracted from the equipment to optical density, a low-pass and high-pass filter at $0.1 \mathrm{~Hz}$ and $0.01 \mathrm{~Hz}$, respectively, were applied. In addition, Channels with a low signal-to-noise ratio $(<30 \mathrm{~dB}$ ) was rejected to exclude unreliable or distorted channels due to environmental noise, and motion artifact removal was performed (Accel threshold, 1.8; Gyro threshold, 1.8; envelope window, 100; motion OD Xcorr, 0.6 ; contact window, 10). Subsequently, the optical density processed in this way was converted to changes in oxygenated $\left(\mathrm{HbO}_{2}\right)$ and deoxygenated $(\mathrm{HHb})$ hemoglobin for each channel using the modified Beer-Lambert law. Although both $\mathrm{HbO}_{2}$ and $\mathrm{HHb}$ could be measured, only $\mathrm{HbO}_{2}$ was measured based on previous findings that $\mathrm{HbO}_{2}$ was more sensitive than $\mathrm{HHb}$ to locomotion-related activities. ${ }^{9}$ Since there was no difference in hemisphere activation (P $>0.05$ ), the values of all channel were summed and averaged. ${ }^{32,33,35}$ For each trial, the 60 -s task duration was divided into a 30-s baseline period, which was measured in quiet standing without any movement as a reference, and a $30 \mathrm{~s}$ task 
period. Hence, we calculated the difference between the baseline and task periods to measure the relative changes in $\mathrm{HbO}_{2} \cdot{ }^{9,33}$ To exclude the subject's anticipatory responses, only the middle 20 s per period was used for data processing, excluding the $5 \mathrm{~s}$ after the start and $5 \mathrm{~s}$ before the end. After one trial (60s) was completed, the subject rested for more than $45 \mathrm{~s}$ to ensure the reduced responsiveness of the PFC activation. ${ }^{8}$ All trials were performed three times, and the results were averaged.

\section{Assessment of gait and cognitive performance}

OptoGait (Microgate Srl, Bolzano, Italy) was used to evaluate gait performance. The gait pattern was analyzed when the subject passed the sensor bars, including light-emitting diodes. ${ }^{37}$ This equipment consists of 10 sensor bars (length 1 $\mathrm{m})$. It can be installed in overground and treadmill walking conditions. In the overground walking assessment, sensor bars were installed on both sides with a length of $5 \mathrm{~m}$ in the center of a 14-m walkway. The participant was instructed to walk back and forth for $30 \mathrm{~s}$ by crossing the sensor bars in the walkway. At the same time, the straight distance $(5 \mathrm{~m})$ before the subject made the first turn was processed as a gait analysis period. ${ }^{38}$ The sensor bars were positioned on both sides of the treadmill belt matching the height of the treadmill belt in which the subject walks for $30 \mathrm{~s} .{ }^{39}$ The analysis processing period on the treadmill excluded the first and last $5 \mathrm{~s}$ to remove acceleration and deceleration phases. The extracted gait parameters in the single-task and dual-task conditions were speed, stride length, gait variability (calculated as [SD stride time/mean stride time] $\times 100 \%$ ), and cadence. Here, low stride time variability represented an indicator of automatic and regular walking and a clinical index of gait stability. ${ }^{40}$ To assess dual-task motor (gait variability, stride length, speed, and cadence) and cognitive performance (total response, $\mathrm{CR}$, and response index), the dual-task cost (DTC) was used as recommended by several studies, ${ }^{19,37,41,42}$ with a lower DTC representing good task performance and lower DTI. The error score was not applied to the DTC because there were cases where errors were not found in a single-task situation. The formula is as follows: DTC = (single-task parameter - dual-task parameter)/single-task parameter $\times 100$, with parameter representing either the motor or cognitive task parameter. For gait variability, where a high value indicates poor gait automaticity, this formula was reversed as follows: DTC $=($ dualtask gait variability - single-task gait variability)/single-task gait variability $\times 100$.

\section{Statistical Analysis}

The Shapiro-Wilk test was used to test the normal distribution. The baseline characteristics between groups were compared using the independent t-test, Mann-Whitney $U$ test, and chi-square test. Values are presented as mean and standard deviation. To explore the effect of age, walking condition, and task factors on PFC activation, we used a $2 \times 2 \times$ 2 mixed-design analysis of variance (ANOVA) with age (older vs. younger adults) as the between-subjects variable and walking condition (overground vs. treadmill) and task (single- vs. dual-task) as the within-subjects variables. We also performed a $2 \times 2$ mixed ANOVA on dual-task performance with age (older vs. younger adults) and walking condition (overground vs. treadmill) as the between-subjects and within-subjects variables, respectively. A Bonferroni post hoc test was conducted to check for significant interaction effects. Furthermore, the correlation between executive function and DTC of dual-task performance was confirmed in various walking conditions using Pearson's correlation analysis. The significance level was set at $p<0.05$. A minimum sample size of 36 subjects was calculated for this study using $G$ * power 3.1, with an effect size of 0.25 and a statistical power of $95 \%$. Based on previous findings with an effect size of 0.96 (Cohen's d), a sample size of 30 subjects was needed, ${ }^{11,17}$ indicating that the study had a larger sample size compared to other studies examining fNIRS data between younger and older adults. ${ }^{11,12,15}$

\section{Results}

\section{Baseline participant characteristics}


Table 1 presents the baseline characteristics of the participants. There were no differences in global cognitive function, cognitive performance under single-task conditions, and gait speed between younger and older adults under single-task and dual-task conditions. However, a significant difference was observed in executive function, as assessed by the TMTB $(P<0.001)$. Additionally, deficits in physical performance, depression, and fear of falling were not found in older adults, as assessed by the SPBB, GDS, and FES-I, with cut-off scores of $\leq 9, \geq 11$, and $>23$, respectively. ${ }^{24-26}$ All older people had no previous falls.

\section{Dual-task performance}

The DTC of gait variability was affected by the condition $(F(1,38)=32.365, P<0.001$, partial $\eta 2=0.461)$ and the interaction between condition and age $(F(1,38)=8.317, P=0.006$, partial $\eta 2=0.180)$. The post-hoc test indicated that the DTC gait variability of older adults during overground walking was higher than that on treadmill walking $(P<0.001$, mean difference, 27.419; 95\% confidence interval [CI], 18.281-36.557) and higher than that of the DTC gait variability in overground walking in younger adults $(P=0.005$, mean difference,18.346; 95\% Cl, 5.757-30.936) (Fig. 2). The condition also exhibited an effect on DTC stride length $(F(1,38)=15.837, P<0.001$, partial $\eta 2=0.294)$ and DTC speed $(F(1,38)=$ $57.464, P<0.001$, partial $\eta 2=0.602$ ). The post-hoc test revealed the values were higher in overground walking than those in treadmill walking $(\mathrm{P}<0.001$, mean difference, 4.045; 95\% Cl, 1.987-6.102; $\mathrm{P}<0.001$, mean difference: 5.508; $95 \% \mathrm{Cl}$ : 4.037-6.979). No main or interaction effects were observed on the cadence. However, the main effects for age were found on DTC of CR $(F(1,38)=8.615, P=0.006$, partial $\eta 2=0.185)$, total response $(F(1,38)=7.324, P=0.010$, partial $\eta 2$ $=0.162)$ and error score $(F(1,38)=4.642, P=0.038$, partial $\eta 2=0.109)$, and for condition were observed in DTC of CR $(F$ $(1,38)=112.121, P<0.001$, partial $\eta 2=0.747)$, total response $(F(1,38)=60.829, P<0.001$, partial $\eta 2=0.615)$, response index $(F(1,38)=7.337, P=0.010$, partial $\eta 2=0.162)$ and error score $(F(1,38)=6.487, P=0.015$, partial $\eta 2=0.146)$. The interaction effects between the two factors were found in the all cognitive variables such as the DTC of $C R(F(1,38)=$ 23.035, $P<0.001$, partial $\eta 2=0.377)$, total response $(F(1,38)=10.421, P=0.003$, partial $\eta 2=0.215)$, response index $(F$ $(1,38)=4.960, P=0.032$, partial $\eta 2=0.115)$, and error score $(F(1,38)=4.795, P=0.035$, partial $\eta 2=0.112)($ Table 2$)$. The post-hoc test revealed that the DTCs and the error score on treadmill walking were lower than that of overground walking for all groups (DTC of CR, $\mathrm{P}<0.001$, mean difference, 31.715; 95\% Cl, 25.815-37.616 and $\mathrm{P}<0.001$, mean difference, 11.932; $95 \% \mathrm{Cl}, 6.031-17.832$, DTC of total response, $\mathrm{P}<0.001$, mean difference, $27.156 ; 95 \% \mathrm{Cl}, 20.106-34.206$ and $\mathrm{P}$ $=0.003$, mean difference, $11.257 ; 95 \% \mathrm{Cl}, 4.207-18.307$ for older and younger adults, respectively, DTC of response index, $\mathrm{P}=0.001$, mean difference, $8.180 ; 95 \% \mathrm{Cl}, 3.435-12.924$, and error score, $\mathrm{P}=0.002$, mean difference, $11.432 ; 95 \%$ $\mathrm{Cl}, 4.522-18.342$ for older adults) and that younger adults had lower DTC of CR ( $<<0.001$, mean difference, $28.444 ; 95 \%$ $\mathrm{Cl}, 14.792-42.095)$, total response $(\mathrm{P}<0.001$, mean difference, 24.069; 95\% Cl, 12.115-36.022), and response index $(P=$ 0.026 , mean difference, $7.106 ; 95 \% \mathrm{Cl}, 0.900-13.312)$, and lower error score ( $\mathrm{P}=0.028$, mean difference, $10.615 ; 95 \% \mathrm{Cl}$, 1.202-20.027) in overground walking compared to older adults (Fig. 2). A positive correlation was observed between the TMTB and DTC in gait variability $(r=0.456 ; P=0.003 ; 95 \% \mathrm{Cl}, 0.185-0.679), \mathrm{CR}(\mathrm{r}=0.549 ; \mathrm{P}<0.001 ; 95 \% \mathrm{Cl}, 0.292-$ $0.771)$, total response $(r=0.517 ; \mathrm{P}=0.001 ; 95 \% \mathrm{Cl}, 0.251-0.739)$, response index $(r=0.437 ; \mathrm{P}=0.005 ; 95 \% \mathrm{Cl}, 0.150-$ 0.656), and error score $(r=0.478 ; \mathrm{P}=0.002 ; 95 \% \mathrm{Cl}, 0.238-0.703)$ in overground walking. In treadmill walking condition, a correlation was observed between TMTB and DTC of stride length only $(r=0.338 ; \mathrm{P}=0.033 ; 95 \% \mathrm{Cl}, 0.045-0.626)$ (Table 3).

\section{PFC activation}

The main effect of condition $(F(1,38)=4.742, P=0.036$, partial $\eta 2=0.111)$ and task $(F(1,38)=18.814, P<0.001$, partial $\eta 2=0.331$ ) was observed on PFC activation. PFC activation during treadmill walking was lower than that during overground walking ( $P=0.036$, mean difference, $0.145 ; 95 \% \mathrm{Cl}, 0.010-0.280)$. Dual-tasking led to higher PFC activation compared to single-tasking ( $\mathrm{P}<0.001$, mean difference, $-0.266 ; 95 \% \mathrm{Cl},-0.390--0.142)$. In addition, the interaction effect of condition by task was found $(F(1,38)=32.515, P<0.001$, partial $\eta 2=0.461)$, The post-hoc test revealed a difference in 
activation between single- and dual-tasking in overground walking $(\mathrm{P}<0.001$, mean difference, $-0.551 ; 95 \% \mathrm{Cl}$, -0.699--0.404) and that PFC activation during the dual-task on overground walking was higher than that of treadmill walking $(P<0.001$, mean difference, $0.430 ; 95 \% \mathrm{Cl}, 0.227-0.584)$. No effect of interaction by age by condition by task was observed. Post-hoc tests revealed that in both older and younger adults, PFC activation during dual-task overground walking was greater than that during single-task overground walking $(\mathrm{P}<0.001$, mean difference, $-0.665 ; 95 \% \mathrm{Cl}$, $-0.873-0.456 ; \mathrm{P}<0.001$, mean difference, $-0.438 ; 95 \% \mathrm{Cl},-0.646--0.229$ for older and younger adults, respectively). The difference in activation between tasks on the treadmill condition was not observed $(P=2.444$, mean difference, -0.142 ; $95 \% \mathrm{Cl},-0.386-0.101 ; \mathrm{P}=0.143$, mean difference, $0.180 ; 95 \% \mathrm{Cl},-0.063-0.423$ for older and younger adults, respectively), but the dual-task activation during treadmill walking was significantly lower than that during overground walking $(\mathrm{P}<$ 0.001 , mean difference, $0.432 ; 95 \% \mathrm{Cl}, 0.215-0.650 ; \mathrm{P}<0.001$, mean difference, $0.428 ; 95 \% \mathrm{Cl}, 0.211-0.645$ for older and younger adults, respectively) (Fig. 3).

\section{Discussion}

To our knowledge, this is the first study to determine and compare PFC activation and DTC during dual-tasking under different conditions (overground vs. treadmill walking) among younger and older adults. Our findings suggest that dualtask treadmill walking leads to lower DTC in motor and cognitive tasks and lower PFC activation compared to dual-task overground walking. Furthermore, differences in DTC in motor and cognitive tasks between older and younger adults, as well as a higher dual-task PFC activation compared to single-task activation, were found only in overground walking, possibly because overground and treadmill walking use different locomotor mechanisms. Specifically, since the treadmill induces a rhythmic movement by maintaining gait automaticity compared to overground, the direct locomotor pathway would have been activated instead of the executive (indirect) locomotor pathway compensating for impaired gait capacity (e.g., challenge condition, dual-tasking). Additionally, executive function (TMTB), which is essential for dualtasking, was correlated with dual-task performance in overground walking compared to treadmill walking.

\section{Dual-task performance}

In older adults, treadmill walking led to improved performance in gait variability, stride length, speed, CR, total response, response index, and error score (lower DTCs and lower error) than overground walking. We also found a high dual-task performance (i.e., stride length, speed, $\mathrm{CR}$, and total response) on treadmill walking than on overground walking in young adults. In addition, the dual-task performance in gait variability, CR, total response, response index, and error score of overground walking in older adults was lower than that in younger adults. Overground walking led to poor dual-task performance (motor and cognitive tasks) compared to treadmill walking, and thus was more sensitive to DTI. ${ }^{19,43}$ Penati et al. ${ }^{18}$ noted that dual-task treadmill walking led to a high cognitive performance than overground walking. Consistent with previous findings by Takeuchi et al., although the DTC in gait and cognitive tasks for older adults was significantly higher than that of younger adults in overground walking, ${ }^{12}$ there was no difference between the two groups in treadmill walking. This is due to the environmental characteristics of the treadmill where externally generated cues (e.g., constant backward treadmill belt) artificially prevent the altered gait pattern caused by DTI, such as decreased speed and stride length, and increase gait automaticity through rhythmic movement. ${ }^{18,37}$ Both gait variability and cognitive performance, which represents automaticity, ${ }^{37,44}$ may be compensated by the influence of the treadmill for older adults; thus, no significant differences between groups would be observed on treadmill walking. This mechanism effect in treadmill walking reduces the attention required for walking during dual-tasking, thereby allocating more attention to the secondary task (cognitive task), leading to superior cognitive processing speed, accuracy, and lower error. ${ }^{18,19}$ Furthermore, correlation analysis revealed that more positive association in overground walking between dual-task performance (gait variability, CR, total response, response index and error) and TMTB relative to treadmill walking (stride length). Executive function, one of the functions of the PFC, plays an important role in dual-tasking, as it is responsible 
for planning, execution, monitoring, inhibition, and attention allocation. ${ }^{45}$ In other words, unlike dual-task overground walking, which is implemented by integrating cognitive and motor skills for the surrounding environment, ${ }^{5,43}$ executive function would have been utilized less in treadmill walking, where automatic gait execution is maintained by passive cue without voluntary control. $8,17,46$ We consider that the role of executive functions is more important in the overground than in the treadmill condition during dual-tasking. Further research is required to address this issue.

\section{PFC activation}

Both older and younger adults had similar patterns in PFC activation in overground and treadmill walking, and they showed high PFC activation during dual-task in overground walking compared to single-task overground walking, revealing similar findings to those of previous studies. ${ }^{47,48}$ However, no difference in PFC activation was found in the treadmill condition, and dual-task treadmill walking showed a lower level of PFC compared to dual-task overground walking. The direct locomotor pathway, where signals of the primary motor cortex are delivered to the spinal network, bypassing the basal ganglia and brainstem locomotor regions, is mainly activated during normal gait or non-challenging situations, requiring relatively less cognitive control. ${ }^{8}$ In contrast, the executive motor pathway, where signals are transmitted through the PFC to the basal ganglia, subthalamic, and mesencephalic locomotor regions, is used to compensate for deficits in gait capacity (e.g., decreased speed and automaticity) in difficult conditions (e.g., dual-task) and pathological and aging conditions. ${ }^{1,8}$ Our findings could be interpreted in terms of the locomotor system based on the mechanism of the treadmill described above, which implied that during dual-task walking, the locomotor pathway system used on the treadmill was different from that in the overground. Stuart et al. ${ }^{17}$ noted that the premotor, supplementary motor, and primary motor areas were activated during dual-task treadmill walking in older people, but with no PFC activation. The PFC was not activated during dual-task treadmill walking in younger adults, ${ }^{11}$ suggesting that during dual-tasking, the activation of the direct locomotor pathway may be predominant in treadmill walking, and the executive motor pathway may be predominant in the overground, which is similar to the results of the correlation analysis. Furthermore, the decreased PFC activity in treadmill could have originated from a transition from attentional demand-gait to more automatic gait, indicating a transition from the indirect to the direct locomotor pathway. ${ }^{14,20}$ However, we observed no difference in the PFC levels between the two groups, possibly because the older people in our study were younger than those in other similar studies (mean age, $<65$ years vs. $\geq 70$ years), ${ }^{48,49}$ indicating less aging. As shown, aging progression was assessed by the TMTB, but the older adults in the present study did not differ from younger adults in terms of global cognitive function, physical function, and gait ability (MoCA, SPPB, and walking speed in single- and dual-task conditions), with the absence of depression and fear of falling. Because the decrease in physical function and gait ability with aging is insufficient to use compensation, there is likely no compensatory PFC overuse compared to younger adults, indicating less advanced aging. Furthermore, age-related over-recruitment in PFC was found in the older adults with advanced aging (77-82 years) compared to those with less advanced aging (61-70 years). ${ }^{50}$ However, although older adults with less advanced aging use a PFC level similar to that in younger adults, older adults showed lower dual-task performance, indicating inefficient use of PFC capacity in terms of behavioral performance, which is consistent with previous findings with differences in performance but no differences in brain activity between groups. ${ }^{11,12,15}$ The finding by Cabeza et al. ${ }^{51}$ showed older adults recruited a similar PFC activation as younger adults but showed lower performance than younger adults during cognitive task due to inefficient neural processing of PFC with aging. Our findings suggest that older adults can maintain the similar level of dual-task performance as younger adults in treadmill walking, with PFC activation reduced. However, although older adults recruit PFC level similar to that in younger adults in overground walking, older adults show lower dual-task performance than younger adults due to inefficient use of PFC with aging. This indicates that treadmill and overground walking do not share similar activation patterns during dual-task walking.

\section{Study limitation}


This study has some limitations. First, it was difficult to generalize the results because of the relatively small sample size, despite the justification of the study. Second, we targeted only PFC measurements according to the study design; however, it is necessary to include measurements from other brain regions for a clearer interpretation, and although the review study recommended calculating and using age-dependent DPF values, we used fixed DPF values due to limitation of analysis tool. However, after the data was processed with the assistance of expert, a visual inspection of the data was also performed once more. Third, only the TMTB was used to evaluate the executive functions. It seems that various cognitive evaluations are needed to evaluate dual-task ability (Trail Making Test A and Stroop Test). Fourth, walking speed is different between walking conditions, ${ }^{43}$ but this study was carried out according to the findings of previous studies that compared overground and treadmill walking. ${ }^{17-19}$ Finally, we did not further assign the older people into different age groups, which is needed to reveal their corresponding aging stages, including less advanced and advanced aging.

\section{Abbreviations}

CR, correct response; DTC, dual-task cost; DTI, dual-task interference; FES-I, Falls Efficacy Scale-International; fNIRS, functional near-infrared spectroscopy; GDS, Geriatric Depression Scale; $\mathrm{HbO}_{2}$, oxygenated hemoglobin; $\mathrm{HHb}$, deoxygenated hemoglobin; MoCA, Montreal Cognitive Assessment; PFC, prefrontal cortex; SPBB, Short Physical Performance Battery; TMTB, Trail Making Test B.

\section{Declarations}

\section{-Ethics approval and consent to participate:}

This study was approved by the National Health Insurance Service of the Ilsan Hospital Institutional Review Board and was conducted in a public hospital in accordance with the Declaration of Helsinki. All participants provided written informed consent before the participation.

-Consent for publication: All authors and participants provided consent for publication

-Availability of data and materials: Data are available upon requesting to the corresponding authors, Chang Yoon Baek, and Hyeong Dong Kim.

-Competing interests: We have no conflicts of interest to disclose.

-Funding: This research did not receive any specific grant from funding agencies in the public, commercial, or not-forprofit sectors.

\section{-Authors' contributions:}

C.Y.B. and H.D.K. devised the main conceptual ideas and designed the study. C.Y.B. and D.Y.Y. performed the experiments. C.Y.B. and D. Y. Y. collected the data. C.Y.B. analyzed the data. C. Y. B. wrote the manuscript. H.D.K. and C.Y.B. commented on and supervised the manuscript. All authors have read and approved the final version of the manuscript.

-Acknowledgements: Not applicable

\section{References}

1. Kahya $\mathrm{M}$, Moon S, Ranchet $\mathrm{M}$, et al. Brain activity during dual task gait and balance in aging and age-related neurodegenerative conditions: a systematic review. Experimental gerontology. 2019;128:110756. 
2. Isingrini $M$, Angel L, Fay S, Taconnat L, Lemaire P, Bouazzaoui B. Age-related differences in the reliance on executive control in working memory: role of task demand. PloS one. 2015;10(12):e0145361.

3. Pelicioni PH, Tijsma M, Lord SR, Menant J. Prefrontal cortical activation measured by fNIRS during walking: effects of age, disease and secondary task. PeerJ. 2019;7:e6833.

4. Wagshul ME, Lucas M, Ye K, Izzetoglu M, Holtzer R. Multi-modal neuroimaging of dual-task walking: Structural MRI and fNIRS analysis reveals prefrontal grey matter volume moderation of brain activation in older adults. Neuroimage. 2019;189:745-754.

5. Fjell AM, Sneve MH, Grydeland $H$, Storsve $A B$, Walhovd KB. The disconnected brain and executive function decline in aging. Cerebral cortex. 2017;27(3):2303-2317.

6. Brustio PR, Magistro D, Zecca M, Rabaglietti E, Liubicich ME. Age-related decrements in dual-task performance: Comparison of different mobility and cognitive tasks. A cross sectional study. PLoS One. 2017;12(7):e0181698.

7. Montero-Odasso MM, Sarquis-Adamson Y, Speechley M, et al. Association of dual-task gait with incident dementia in mild cognitive impairment: results from the gait and brain study. JAMA neurology. 2017;74(7):857-865.

8. Herold F, Wiegel P, Scholkmann F, Thiers A, Hamacher D, Schega L. Functional near-infrared spectroscopy in movement science: a systematic review on cortical activity in postural and walking tasks. Neurophotonics. 2017;4(4):041403.

9. Nóbrega-Sousa P, Gobbi LTB, Orcioli-Silva D, Conceição NRd, Beretta VS, Vitório R. Prefrontal cortex activity during walking: effects of aging and associations with gait and executive function. Neurorehabilitation and Neural Repair. 2020;34(10):915-924.

10. Salzman T, Tobón Vallejo D, Polskaia N, et al. Hemodynamic and behavioral changes in older adults during cognitively demanding dual tasks. Brain and behavior. 2020:e02021.

11. Beurskens R, Helmich I, Rein R, Bock O. Age-related changes in prefrontal activity during walking in dual-task situations: a fNIRS study. International journal of psychophysiology. 2014;92(3):122-128.

12. Takeuchi N, Mori T, Suzukamo Y, Tanaka N, Izumi S-I. Parallel processing of cognitive and physical demands in left and right prefrontal cortices during smartphone use while walking. BMC neuroscience. 2016;17(1):1-11.

13. Al-Yahya E, Johansen-Berg H, Kischka U, Zarei M, Cockburn J, Dawes H. Prefrontal cortex activation while walking under dual-task conditions in stroke: a multimodal imaging study. Neurorehabilitation and neural repair. 2016;30(6):591-599.

14. Eggenberger P, Wolf M, Schumann M, de Bruin ED. Exergame and Balance Training Modulate Prefrontal Brain Activity during Walking and Enhance Executive Function in Older Adults. Frontiers in Aging Neuroscience. 2016;8.

15. Fraser SA, Dupuy O, Pouliot P, Lesage F, Bherer L. Comparable cerebral oxygenation patterns in younger and older adults during dual-task walking with increasing load. Frontiers in aging neuroscience. 2016;8:240.

16. Springer S, Gottlieb U. Effects of dual-task and walking speed on gait variability in people with chronic ankle instability: a cross-sectional study. BMC musculoskeletal disorders. 2017;18(1):1-8.

17. Stuart S, Alcock L, Rochester L, Vitorio R, Pantall A. Monitoring multiple cortical regions during walking in young and older adults: Dual-task response and comparison challenges. International journal of psychophysiology. 2019;135:63-72.

18. Penati R, Schieppati $M$, Nardone A. Cognitive performance during gait is worsened by overground but enhanced by treadmill walking. Gait \& posture. 2020;76:182-187.

19. Wrightson JG, Schäfer L, Smeeton NJ. Dual-task prioritization during overground and treadmill walking in healthy adults. Gait \& posture. 2020;75:109-114.

20. Thumm PC, Maidan I, Brozgol M, et al. Treadmill walking reduces pre-frontal activation in patients with Parkinson's disease. Gait \& posture. 2018;62:384-387. 
21. Lee JY, Dong Woo L, Cho SJ, et al. Brief screening for mild cognitive impairment in elderly outpatient clinic: validation of the Korean version of the Montreal Cognitive Assessment. Journal of geriatric psychiatry and neurology. 2008;21(2):104-110.

22. Kortte KB, Horner MD, Windham WK. The trail making test, part B: cognitive flexibility or ability to maintain set? Applied neuropsychology. 2002;9(2):106-109.

23. Montero-Odasso M, Bergman $\mathrm{H}$, Phillips NA, Wong $\mathrm{CH}$, Sourial N, Chertkow H. Dual-tasking and gait in people with mild cognitive impairment. The effect of working memory. BMC Geriatr. 2009;9:41.

24. Pritchard J, Kennedy C, Karampatos S, et al. Measuring frailty in clinical practice: a comparison of physical frailty assessment methods in a geriatric out-patient clinic. BMC geriatrics. 2017;17(1):1-8.

25. Smarr KL, Keefer AL. Measures of depression and depressive symptoms: beck depression inventory-II (BDI-II), Center for Epidemiologic Studies Depression Scale (CES-D), geriatric depression scale (GDS), hospital anxiety and depression scale (HADS), and patient health Questionnaire-9 (PHQ-9). Arthritis care \& research. 2011;63(S11):S454S466.

26. Marques-Vieira CMA, Sousa LMM, Severino S, Sousa L, Caldeira S. Cross-cultural validation of the falls efficacy scale international in elderly: systematic literature review. Journal of Clinical Gerontology and Geriatrics. 2016;7(3):72-76.

27. Granacher U, Wolf I, Wehrle A, Bridenbaugh S, Kressig RW. Effects of muscle fatigue on gait characteristics under single and dual-task conditions in young and older adults. Journal of neuroengineering and rehabilitation. 2010;7(1):1-12.

28. Hobert MA, Meyer SI, Hasmann SE, et al. Gait is associated with cognitive flexibility: a dual-tasking study in healthy older people. Frontiers in aging neuroscience. 2017;9:154.

29. Manor B, Zhou J, Jor'dan A, Zhang J, Fang J, Pascual-Leone A. Reduction of dual-task costs by noninvasive modulation of prefrontal activity in healthy elders. Journal of cognitive neuroscience. 2016;28(2):275-281.

30. Fino PC, Mancini M, Curtze C, Nutt JG, Horak FB. Gait stability has phase-dependent dual-task costs in Parkinson's disease. Frontiers in neurology. 2018;9:373.

31. Dong S-Y, Choi J, Park Y, et al. Prefrontal Functional Connectivity During the Verbal Fluency Task in Patients With Major Depressive Disorder: A Functional Near-Infrared Spectroscopy Study. Frontiers in psychiatry. 2021;12:674.

32. Hawkins KA, Fox EJ, Daly JJ, et al. Prefrontal over-activation during walking in people with mobility deficits: interpretation and functional implications. Human movement science. 2018;59:46-55.

33. Mirelman A, Maidan I, Bernad-Elazari $\mathrm{H}$, et al. Increased frontal brain activation during walking while dual tasking: an fNIRS study in healthy young adults. Journal of neuroengineering and rehabilitation. 2014;11(1):1-7.

34. Lee B-C, Choi J, Martin BJ. Roles of the prefrontal cortex in learning to time the onset of pre-existing motor programs. PloS one. 2020;15(11):e0241562.

35. Yu J-W, Lim S-H, Kim B, et al. Prefrontal functional connectivity analysis of cognitive decline for early diagnosis of mild cognitive impairment: a functional near-infrared spectroscopy study. Biomedical optics express. 2020;11(4):1725-1741.

36. Menant JC, Maidan I, Alcock L, et al. A consensus guide to using functional near-infrared spectroscopy in posture and gait research. Gait \& posture. 2020.

37. Baek CY, Chang WN, Park BY, Lee KB, Kang KY, Choi MR. Effects of dual-task gait treadmill training on gait ability, dual-task interference, and fall efficacy in people with stroke: A Randomized Controlled Trial. Physical therapy. 2021;101(6):pzab067.

38. Liu Y-C, Yang Y-R, Tsai Y-A, Wang R-Y, Lu C-F. Brain activation and gait alteration during cognitive and motor dual task walking in stroke-a functional near-infrared spectroscopy study. IEEE transactions on neural systems and 
rehabilitation engineering. 2018;26(12):2416-2423.

39. Lee M, Song C, Lee K, Shin D, Shin S. Agreement between the spatio-temporal gait parameters from treadmill-based photoelectric cell and the instrumented treadmill system in healthy young adults and stroke patients. Medical science monitor: international medical journal of experimental and clinical research. 2014;20:1210.

40. Beauchet $\mathrm{O}$, Annweiler C, Lecordroch Y, et al. Walking speed-related changes in stride time variability: effects of decreased speed. Journal of neuroengineering and rehabilitation. 2009;6(1):1-6.

41. Baek CY, Yoon HS, Kim HD, Kang KY. The effect of the degree of dual-task interference on gait, dual-task cost, cognitive ability, balance, and fall efficacy in people with stroke: A cross-sectional study. Medicine. 2021;100(24):e26275.

42. Plummer P, Eskes G. Measuring treatment effects on dual-task performance: a framework for research and clinical practice. Frontiers in human neuroscience. 2015;9:225.

43. Simoni D, Rubbieri G, Baccini M, et al. Different motor tasks impact differently on cognitive performance of older persons during dual task tests. Clinical biomechanics. 2013;28(6):692-696.

44. Montero-Odasso M, Muir SW, Hall M, et al. Gait variability is associated with frailty in community-dwelling older adults. Journals of Gerontology Series A: Biomedical Sciences and Medical Sciences. 2011;66(5):568-576.

45. Parikh $\mathrm{H}$, Shah $\mathrm{C}$. Relationship between executive function and dual task physical performance among older adultsa cross sectional study. Int J Phys Med Rehabil. 2017;5(421):10.4172.

46. Sousa AVCd, Santiago LMdM, Silva REdOd, Oliveira DAd, Galvão ÉRVP, Lindquist ARR. Influence of treadmill training in dual-task gait in people with Parkinson's Disease: a case report. Fisioterapia e Pesquisa. 2014;21(3):291-296.

47. Holtzer R, Mahoney JR, Izzetoglu M, Izzetoglu K, Onaral B, Verghese J. fNIRS study of walking and walking while talking in young and old individuals. Journals of Gerontology Series A: Biomedical Sciences and Medical Sciences. 2011;66(8):879-887.

48. Mirelman A, Maidan I, Bernad-Elazari H, Shustack S, Giladi N, Hausdorff JM. Effects of aging on prefrontal brain activation during challenging walking conditions. Brain and cognition. 2017;115:41-46.

49. Ohsugi H, Ohgi S, Shigemori K, Schneider EB. Differences in dual-task performance and prefrontal cortex activation between younger and older adults. BMC neuroscience. 2013;14(1):1-9.

50. Suzuki M, Kawagoe T, Nishiguchi S, et al. Neural correlates of working memory maintenance in advanced aging: evidence from fMRI. Frontiers in Aging Neuroscience. 2018:358.

51. Cabeza R, Anderson ND, Locantore JK, Mclntosh AR. Aging gracefully: compensatory brain activity in highperforming older adults. Neuroimage. 2002;17(3):1394-1402.

\section{Tables}

Table 1. Baseline characteristics of participants 


\begin{tabular}{|c|c|c|c|}
\hline Baseline characteristic & $\begin{array}{l}\text { Older } \\
\text { adults }(N=20)\end{array}$ & Younger adults $(\mathrm{N}=20)$ & $\mathbf{P}$ \\
\hline Age, years & $64.35 \pm 2.74$ & $30.00 \pm 3.15$ & \\
\hline \multicolumn{4}{|l|}{ Sex } \\
\hline Male/Female & $8 / 12$ & $13 / 7$ & 0.113 \\
\hline \multicolumn{4}{|l|}{ Global cognitive function } \\
\hline MoCA score ${ }^{a}$ & $27.00 \pm 1.75$ & $29.00 \pm 2.00$ & 0.053 \\
\hline \multicolumn{4}{|l|}{ Executive function } \\
\hline TMTB, s & $110.43 \pm 24.81$ & $49.30 \pm 12.04$ & $<0.001$ \\
\hline \multicolumn{4}{|c|}{ Single-task cognitive performance } \\
\hline Correct response, $\mathrm{n}$ & $11.05 \pm 3.97$ & $13.10 \pm 3.93$ & 0.065 \\
\hline Total response, $\mathrm{n}$ & $11.38 \pm 3.91$ & $13.42 \pm 3.82$ & 0.103 \\
\hline Response index, \% & $13.57 \pm 0.70$ & $13.61 \pm 0.61$ & 0.911 \\
\hline Error response, $\mathrm{n}$ & $0.33 \pm 0.56$ & $0.32 \pm 0.57$ & 0.812 \\
\hline Single-task gait speed, m/s & $1.26 \pm 0.15$ & $1.25 \pm 0.13$ & 0.929 \\
\hline \multicolumn{3}{|l|}{ speed, m/s } & 0.899 \\
\hline SPPB score & $11.95 \pm 0.22$ & NA & NA \\
\hline \multicolumn{4}{|l|}{ Depression } \\
\hline GDS score & $4.75 \pm 2.00$ & NA & NA \\
\hline \multicolumn{4}{|l|}{ Falling factor } \\
\hline FES-I score & $16.95 \pm 5.49$ & NA & NA \\
\hline \multicolumn{4}{|l|}{ Fall within 6 months } \\
\hline Yes/No & $0 / 20$ & NA & NA \\
\hline
\end{tabular}

FES-I, Falls Efficacy Scale-International; GDS, Geriatric Depression Scale; MoCA, Montreal Cognitive Assessment; SPPB, Short Physical Performance Battery; TMTB, Trail Making Test B, ${ }^{\text {a }}$, applied by Mann-Whitney U test.

Table 2. Comparison of dual task performance 


\begin{tabular}{|c|c|c|c|c|c|c|c|}
\hline & \multicolumn{2}{|l|}{$\begin{array}{l}\text { Older adults } \\
(\mathrm{N}=20)\end{array}$} & \multicolumn{2}{|l|}{$\begin{array}{l}\text { Younger adults } \\
(\mathrm{N}=20)\end{array}$} & \multicolumn{2}{|c|}{ Main effect } & \multirow{2}{*}{$\begin{array}{l}\text { Interaction } \\
\text { effect } \\
\text { condition } \\
\text { by age }\end{array}$} \\
\hline & $\begin{array}{l}\text { Over-ground } \\
\text { walking }\end{array}$ & $\begin{array}{l}\text { Treadmill } \\
\text { walking }\end{array}$ & $\begin{array}{l}\text { Over-ground } \\
\text { walking }\end{array}$ & $\begin{array}{l}\text { Treadmill } \\
\text { walking }\end{array}$ & condition & age & \\
\hline \multicolumn{8}{|l|}{$\begin{array}{l}\text { DTC in } \\
\text { motor } \\
\text { task }\end{array}$} \\
\hline $\begin{array}{l}\text { Gait } \\
\text { variability, } \\
\%\end{array}$ & $36.70 \pm 22.49$ & $9.28 \pm 15.88^{a}$ & $18.35 \pm 16.36^{d}$ & $9.34 \pm 14.54$ & $<0.001$ & 0.052 & 0.006 \\
\hline Stride, \% & $5.04 \pm 3.55$ & $2.49 \pm 6.81^{b}$ & $1.00 \pm 3.92$ & $-1.56 \pm 5.95^{b}$ & $<0.001$ & 0.058 & 0.992 \\
\hline Speed, \% & $7.47 \pm 5.47$ & $0.67 \pm 1.62^{\mathrm{a}}$ & $4.99 \pm 2.81$ & $0.77 \pm 1.57^{a}$ & $<0.001$ & 0.116 & 0.085 \\
\hline $\begin{array}{l}\text { Cadence, } \\
\%\end{array}$ & $3.01 \pm 4.87$ & $1.64 \pm 8.62$ & $4.65 \pm 3.30$ & $2.09 \pm 3.37$ & 0.109 & 0.408 & 0.623 \\
\hline \multicolumn{8}{|l|}{$\begin{array}{l}\text { DTC in } \\
\text { cognitive } \\
\text { task }\end{array}$} \\
\hline $\begin{array}{l}\text { Correct } \\
\text { response, } \\
\%\end{array}$ & $32.51 \pm 24.11$ & $0.80 \pm 23.63^{a}$ & $4.07 \pm 18.12^{c}$ & $-7.86 \pm 17.32^{\mathrm{a}}$ & $<0.001$ & 0.006 & $<0.001$ \\
\hline $\begin{array}{l}\text { Total } \\
\text { response, } \\
\%\end{array}$ & $27.72 \pm 21.16$ & $0.56 \pm 26.78^{a}$ & $3.65 \pm 15.18^{c}$ & $-7.61 \pm 15.72^{b}$ & $<0.001$ & 0.010 & 0.003 \\
\hline $\begin{array}{l}\text { Response } \\
\text { index, \% }\end{array}$ & $7.71 \pm 12.82$ & $-0.47 \pm 6.07^{b}$ & $0.60 \pm 4.87^{e}$ & $-0.20 \pm 5.62$ & 0.010 & 0.082 & 0.032 \\
\hline $\begin{array}{l}\text { Error } \\
\text { score, \% }\end{array}$ & $14.48 \pm 19.65$ & $3.05 \pm 4.28^{b}$ & $3.87 \pm 6.79^{\mathrm{e}}$ & $3.00 \pm 5.21$ & 0.015 & 0.038 & 0.035 \\
\hline
\end{tabular}

DTC, dual task cost, ${ }^{a} p<0.001$, indicating a significant difference between overground and treadmill walking, ${ }^{b} p<0.01$, indicating a significant difference between overground and treadmill walking, ${ }^{c} p<0.001$, indicating a significant difference between groups in overground walking, ${ }^{d} p<0.01$, indicating a significant difference between groups in overground walking, ${ }^{e} p<0.05$, indicating a significant difference between groups in overground walking.

Table 3. Relationship between dual task performance and TMTB 
Dual task performance in overground Dual task performance in treadmill

\begin{tabular}{lllllll}
\hline DTC in variables & $r$ & P-value & $95 \% \mathrm{Cl}$ & $\mathrm{r}$ & P-value & $95 \% \mathrm{Cl}$ \\
\hline Gait variability, \% & 0.456 & 0.003 & $0.185,0.679$ & -0.020 & 0.902 & $-0.339,0.229$ \\
\hline Stride, \% & 0.238 & 0.139 & $-0.051,0.483$ & 0.338 & 0.033 & $0.045,0.626$ \\
\hline Speed, \% & 0.284 & 0.076 & $-0.066,0.662$ & -0.126 & 0.440 & $-0.418,0.201$ \\
\hline Cadence, \% & -0.167 & 0.303 & $-0.448,0.160$ & -0.097 & 0.553 & $-0.493,0.292$ \\
\hline Correct response, \% & 0.549 & $<0.001$ & $0.292,0.771$ & 0.217 & 0.179 & $-0.125,0.533$ \\
\hline Total answer \% & 0.517 & 0.001 & $0.251,0.739$ & 0.213 & 0.187 & $-0.124,0.558$ \\
\hline Response index, \% & 0.437 & 0.005 & $0.150,0.656$ & -0.077 & 0.636 & $-0.355,0.196$ \\
\hline Error score, \% & 0.478 & 0.002 & $0.238-0.703$ & 0.104 & 0.523 & $-0.145,0.416$
\end{tabular}

DTC, dual task cost, TMTB, Trail Making Test B.

\section{Figures}

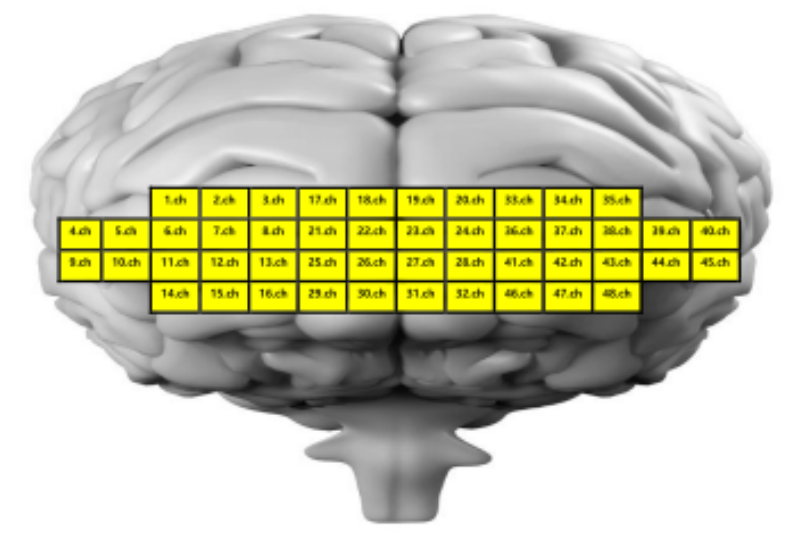

\section{Figure 1}

Arrangement of channels in PFC. The numbers represent channels. Ch, channel. 
a)

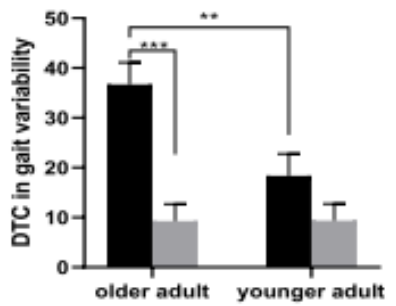

d)

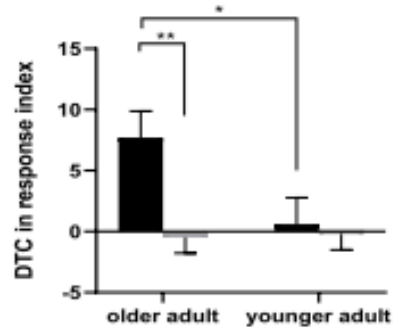

b)

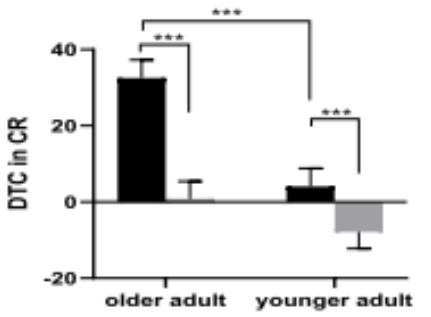

c)

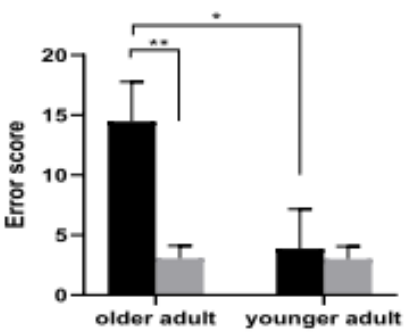

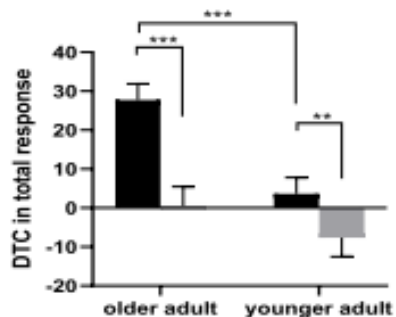

meverground

treadmill

Figure 2

Comparison of DTC in dual-task performance (Mean \pm SE). (a) DTC in gait variability. (b) DTC in CR. (c) DTC in total response. (d) DTC in response index. (e) Error score. CR, correct response; DTC, dual-task cos, * $P<0.05, * \star, P<0.01$, ***, $P<0.001$.

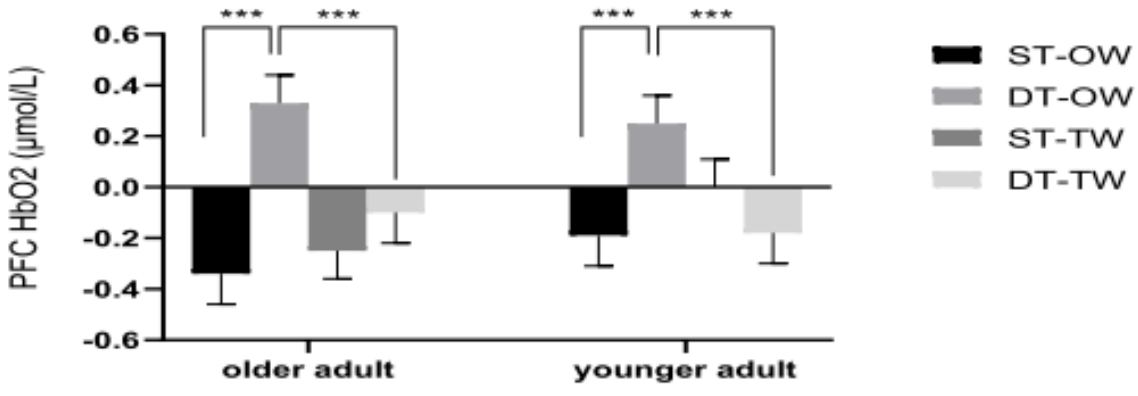




\section{Figure 3}

Comparison of PFC cortex activation (Mean $\pm \mathrm{SE}$ ). PFC, prefrontal cortex; $\mathrm{HbO}_{2}$, oxygenated hemoglobin; ST, single-task; DT, dual-task; OW, overground walking; TW, treadmill walking; ***, $\mathrm{P}<0.001$. 